\title{
Left Basal Anterior Fibromuscular Stroma of Prostate
}

National Cancer Institute

\section{Source}

National Cancer Institute. Left Basal Anterior Fibromuscular Stroma of Prostate. NCI

Thesaurus. Code C128586.

The portion of the fibromuscular stroma of the prostate that is located on the anatomical left side of the basal division of the anterior lobe. 